\title{
Assessment of the Accuracy of the Multiple-Relaxation-Time Lattice Boltzmann Method for the Simulation of Circulating Flows
}

\author{
Mohammed Ahmed Boraey \\ Mechanical Power Engineering Department, Faculty of Engineering, Zagazig University, Zagazig, Egypt \\ Email address: \\ maboraey@zu.edu.eg \\ To cite this article: \\ Mohammed Ahmed Boraey. Assessment of the Accuracy of the Multiple-Relaxation-Time Lattice Boltzmann Method for the Simulation of \\ Circulating Flows. Mathematical Modelling and Applications. Vol. 2, No. 5, 2017, pp. 47-51. doi: 10.11648/j.mma.20170205.11
}

Received: September 23, 2017; Accepted: October 24, 2017; Published: November 20, 2017

\begin{abstract}
The present work investigates the accuracy of the Multiple-relaxation-time Lattice Boltzmann Method (MRT LBM) in the simulation of flows with circulation. The flow in a 2Dlid-driven cavity is simulated using MRT LBM for a wide range of Reynolds numbers (100-1000) to assess its accuracy. The lid-driven cavity flow is selected because it is the standard benchmark problem for the testing of numerical methods. The calculated locations of the primary vortex center in addition to those of the two side vortices (lower-left and lower-right) are compared to the previously published results using different numerical techniques such as finite difference, finite element and single-relaxation-time LBM. The horizontal and vertical velocity profiles are also calculated. The results show that the MRT LBM has a superior accuracy compared to other numerical techniques especially for circulating flows.
\end{abstract}

Keywords: Multiple-Relaxation-Time, Numerical Accuracy, Lattice Boltzmann Method, Circulating Flow, Lid-Driven Cavity Flow

\section{Introduction}

Computational fluid dynamics (CFD) numerical techniques has changed and varied dramatically in the past few years [1]. The reason for this is the diversity in the applications of the CFD [2-6]. Although continuum based CFD approaches like finite difference, finite volume and finite element relay on the solution of continuum governing equations (i.e. macro scale), other emerging techniques are looking at the same problems from a different scale. These can range from the micro-scale based methods like molecular dynamics to macro-scale ones like finite volume.

The Lattice Boltzmann Method (LBM) has emerged as a numerical technique dealing with the problems at the mesoscale [7]. This allowed the LBM to retain the advantages of the two scales (i.e. macro and micro) while avoiding their short comings. The LBM has been widely used for many applications including fluid flow [7,8], heat and mass transfer [9], multi-phase [10] and multi-component [11], nonNewtonian fluids $[12,13]$, fluid-structure interaction [14] and flow in porous media $[12,15]$ problems. The LBM has many variants according to the target application. The tworelaxation-time (TRT) LBM and multiple-relaxation-time (MRT) LBM were invented to overcome the shortcomings of the standard single-relaxation-time (SRT) LBM [16-19].

For each emerging new computational technique, a set of benchmark cases has to be used to test the new model accuracy. The lid-driven cavity flow is the most widely used case for benchmarking numerical methods [20-23]. It involves high velocity gradients and strain rates in addition to circulation. Circulating flow is encountered in many applications and its modeling is more challenging than the unidirectional flow [24, 25]. For this reason, new numerical methods have to be tested for their accuracy using the liddriven cavity flow case before being used for applications involving circulation.

The main goal of the present work is to assess the accuracy of the MR TLBM in modeling circulating flow. This is achieved by simulating the flow inside a 2D lid-driven cavity at a wide range of Reynolds numbers and comparing the results to the published ones in literature. 


\section{TheMultiple-Relaxation-Time LBM}

The standard LBM relies on solving the Boltzmann equation in a discretized form using a limited set of velocity directions. The discrete Boltzmann equation can be written as follows:

$$
f_{i}\left(x_{i}+c \Delta t, t+\Delta t\right)-f\left(x_{i}, t\right)=\Omega(f)
$$

$f_{i}$ is the particle distribution function along directioni,cis the lattice speed $c=\Delta x / \Delta t$ and $\Omega(f)$ is the collision operator. For the used $D 2 Q 9$ lattice the directional velocities $c_{i}$ are given by:

$$
c_{i}=\left\{\begin{array}{cl}
(0,0) & i=0 \\
( \pm 1,0) c,(0, \pm 1) c & i=1: 4 \\
( \pm 1, \pm 1) c & i=5: 8
\end{array}\right.
$$

For the SRT LBM $\Omega(f)$ is replaced by the BhatnagarGross-Krook (BGK) collision operator [26]. Due to many limitation of the standard SRT LBM, the MRT LBM is used instead. In the MRT LBM the collision operator is expressed as follows:

$$
\Omega(f)=-M^{-1} \cdot S \cdot\left[m-m^{e q}\right]
$$

$M$ is a transformation matrix to transform the particle distribution function $f$ from the velocity space to the moment space $m=M$. $f$. The equilibrium distribution function $f^{e q}$ is also transformed to $m^{e q}=M . f^{e q} . S$ is the diagonal relaxation matrix.

$$
\begin{gathered}
M=\left[\begin{array}{ccccccccc}
1 & 1 & 1 & 1 & 1 & 1 & 1 & 1 & 1 \\
-4 & -1 & -1 & -1 & -1 & 2 & 2 & 2 & 2 \\
4 & -2 & -2 & -2 & -2 & 1 & 1 & 1 & 1 \\
0 & 1 & 0 & -1 & 0 & 1 & -1 & -1 & 1 \\
0 & -2 & 0 & 2 & 0 & 1 & -1 & -1 & 1 \\
0 & 0 & 1 & 0 & -1 & 1 & 1 & -1 & 1 \\
0 & 0 & -2 & 0 & 2 & 1 & 1 & -1 & -1 \\
0 & 1 & -1 & 1 & -1 & 0 & 0 & 0 & 0 \\
0 & 0 & 0 & 0 & 0 & 1 & -1 & 1 & -1
\end{array}\right] \\
S=\operatorname{diag}\left(0, s_{1}, s_{2}, 0, s_{4}, 0, s_{6}, s_{v}, s_{v}\right)
\end{gathered}
$$

For the used $D 2 Q 9$ lattice, the sonic speed is given by:

$$
c_{s}=\frac{c}{\sqrt{3}}
$$

The kinematic viscosity $v$ is related to $s_{v}$ by the following relation:

$$
v=c_{s}^{2}\left(\frac{1}{s_{v}}-\frac{1}{2}\right)
$$
by:

The equilibrium particle distribution function $f^{e q}$ is given

$$
f_{i}^{e q}=w_{i} \rho\left[1+\frac{c_{i} \cdot u}{c_{s}^{2}}+\frac{\left(c_{i} \cdot u\right)^{2}}{2 c_{s}^{4}}-\frac{u \cdot u}{2 c_{s}^{2}}\right]
$$

And the macroscopic density $\rho$ and velocity $u$ are given by:

$$
\begin{aligned}
\rho(x, t) & =\sum_{i} f_{i}(x, t) \\
u_{j}(x, t) & =\frac{1}{\rho(x, t)} \sum_{i} c_{i j} f_{i}(x, t)
\end{aligned}
$$

\section{The Lid-Driven Cavity Flow}

The lid-driven cavity flow case is used as a benchmark problem to test the accuracy of the MRT LBM. The modeled geometry shown in Figure 1 consists of a square cavity with the top side moving to the right. The rest of the sides are stationary. The movement of the top surface causes a vortex to develop inside the cavity whose size, strength and location depend on the flow Reynolds number. At the two lower corners (left and right) two small vertices with a circulation in an opposite direction to that of the main vortex also develop. Their characteristics also depend on the flow Reynolds number. The location of the three vortices centers is used as a benchmark for the accuracy of the numerical method used to solve the problem.

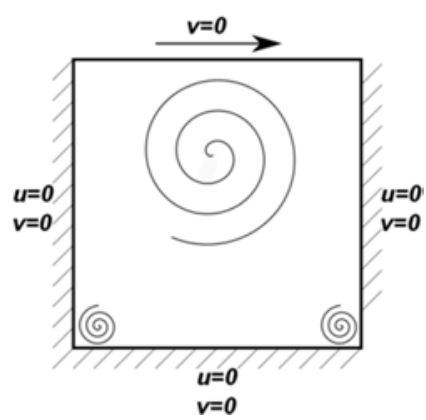

Figure 1. The lid-driven cavity geometry and boundary conditions.

\section{Results and Discussion}

The MRT LBM is used in the simulation of steady viscous incompressible flow in a 2D lid-driven cavity at a range of Reynolds number between 100 and 1000. The flow Reynolds number is calculated based on the cavity width and the velocity of the top lid as a characteristic velocity. The streamlines for some of the cases are shown in Figure 2.

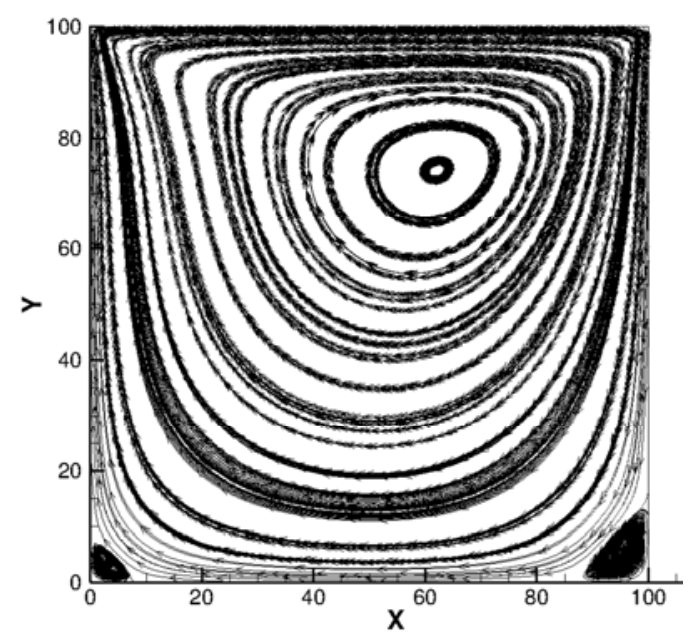

(a) $\operatorname{Re}=100$. 


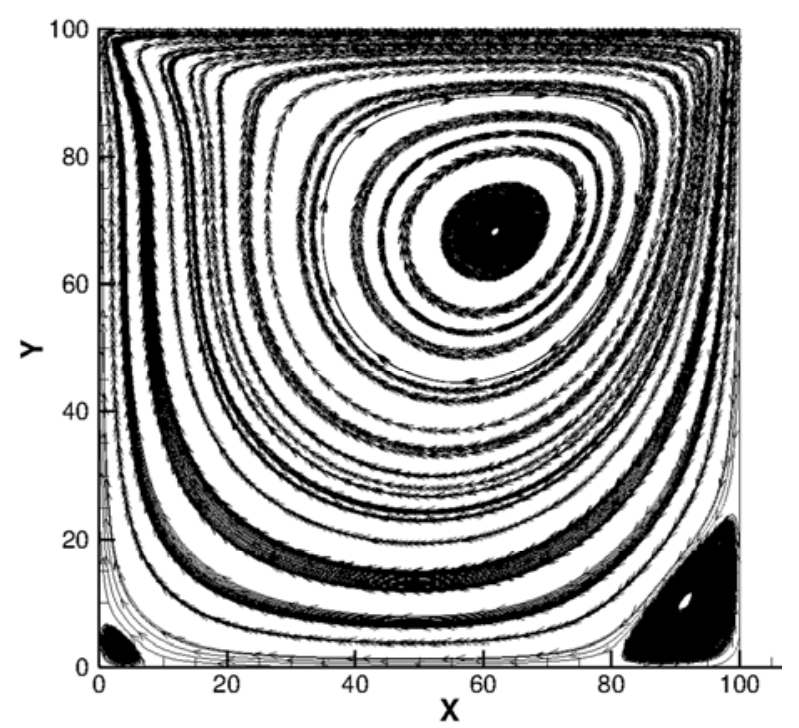

(b) $\operatorname{Re}=200$.

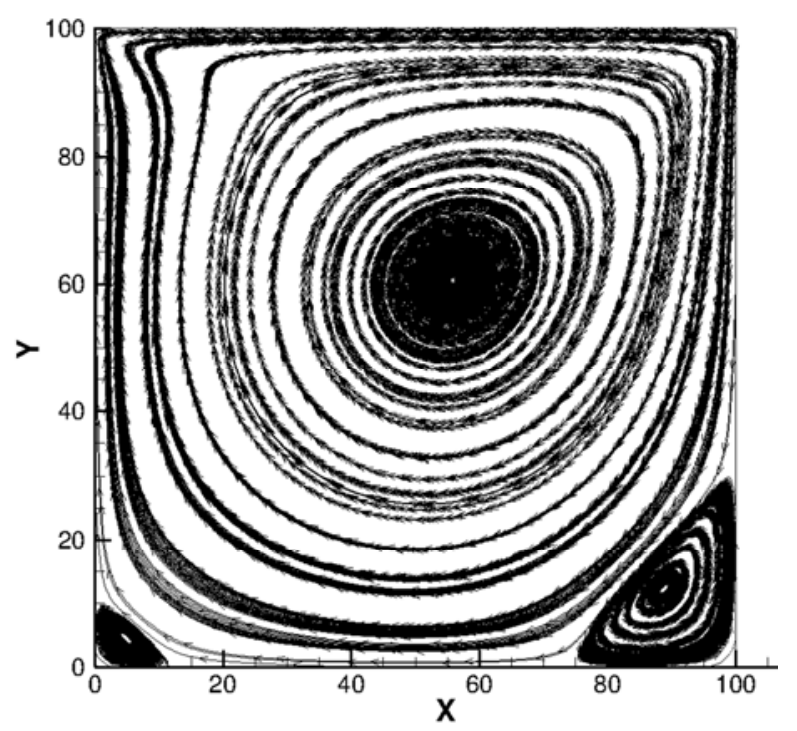

(c) $\mathrm{Re}=400$

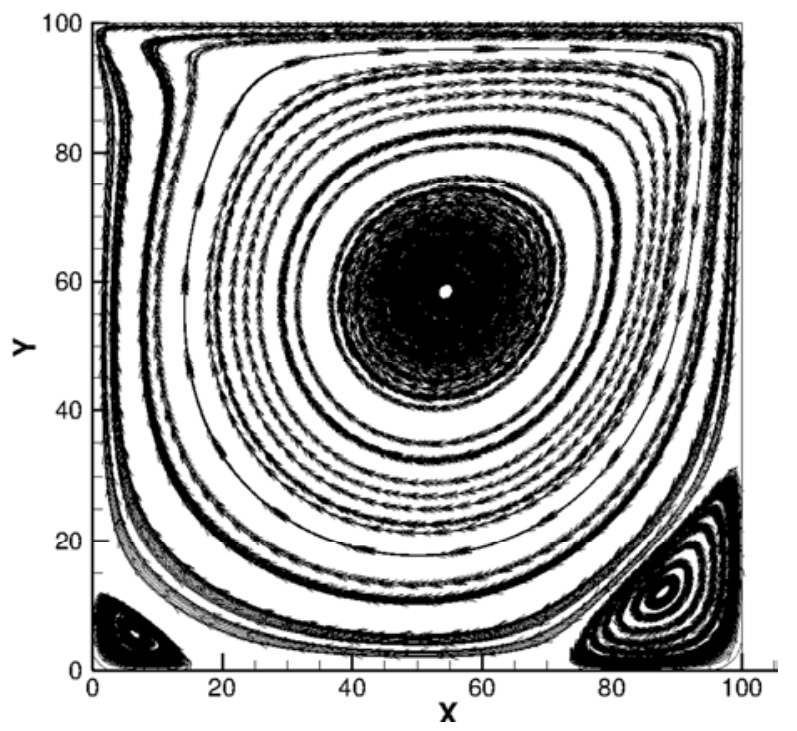

(d) $\operatorname{Re}=600$.

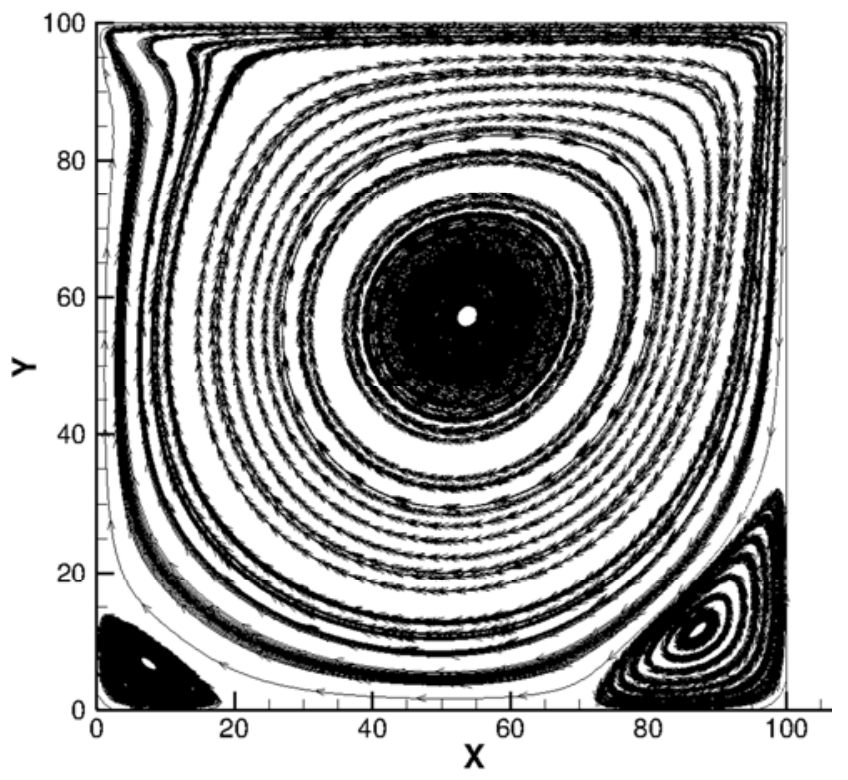

(e) $\operatorname{Re}=800$

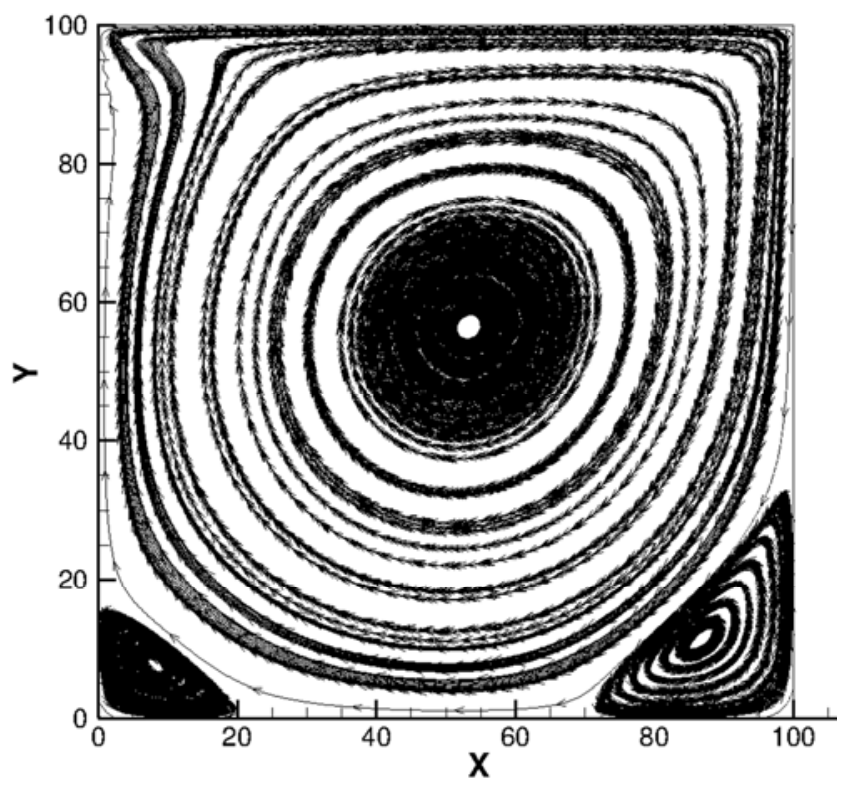

(f) $\operatorname{Re}=1000$.

Figure 2. The steady state streamlines for different $R e=100,200,400,600$, $800 \& 1000$.

The figure shows the variation in the size and location of the vortices with the Reynolds number. The main vortex tends to move to the center of the cavity with the increase in $R e$ number while the two side vortices increase in size. The streamlines at the top of the cavity also show a high gradient of the tangential velocity which is a characteristic of the liddriven cavity flow. Tangential velocity profiles at $x=50$ for different Reynolds numbers are shown in Figure 3. The figure confirms the high gradient of the tangential velocity component near the top lid region. 


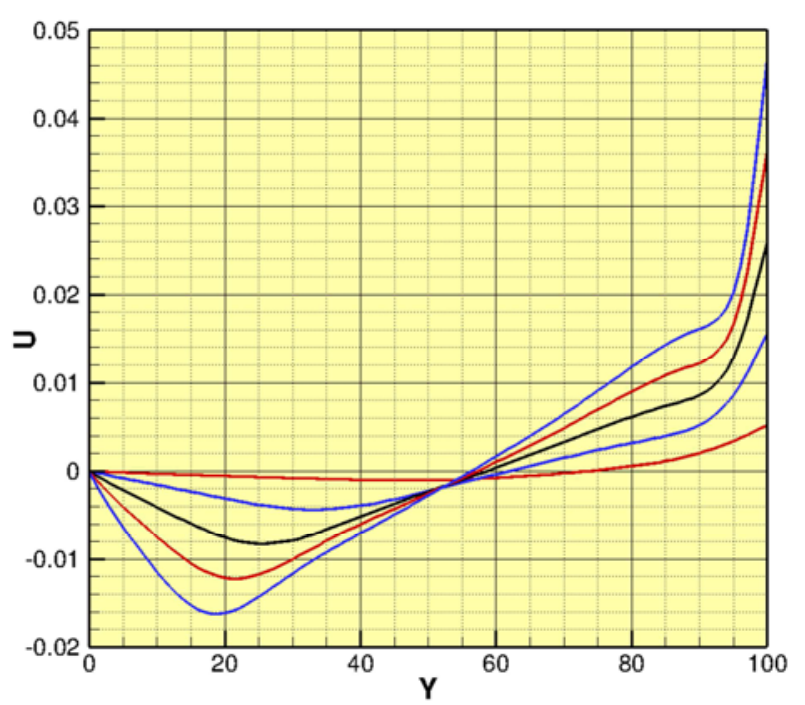

Figure 3. Tangential velocity profiles at $x=50$. Starting at the red semihorizontal line for $R e=100,300,500,700 \& 900$.

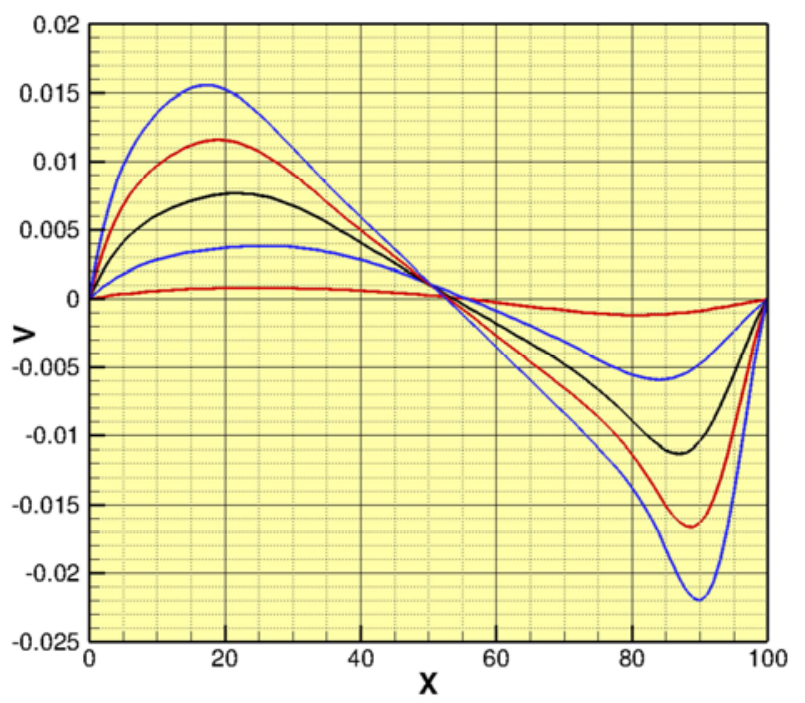

Figure 4. Vertical velocity profiles at $y=50$. Starting at the red semihorizontal line for $R e=100,300,500,700 \& 900$.

The vertical velocity component profiles at $\boldsymbol{y}=\mathbf{5 0}$ are shown in Figure 4.

To test the accuracy of the simulation results, the location of the main vortex and the two side vortices are compared against published results. Table 1 shows this comparison for selected Reynolds numbers.

Table 1. The location of the primary vortex and the two side vortices for $R e=100 \& 1000$ using the MRT LBM (present) compared to published results (Ref. [27-31]).

\begin{tabular}{lllll}
\hline Re & $\mathbf{1 0 0}$ & \multicolumn{3}{l}{$\mathbf{1 0 0 0}$} \\
\hline Primary & $\mathbf{X}$ & $\mathbf{Y}$ & $\mathbf{X}$ & $\mathbf{Y}$ \\
\hline$[27]$ & 0.6188 & 0.7375 & 0.5438 & 0.5625 \\
{$[28]$} & 0.6172 & 0.7344 & 0.5313 & 0.5625 \\
{$[29]$} & 0.6196 & 0.7373 & 0.5333 & 0.5647 \\
{$[30]$} & 0.6167 & 0.7417 & 0.5286 & 0.5643 \\
{$[31]$} & 0.6125 & 0.7375 & 0.5250 & 0.5625 \\
Present & 0.6210 & 0.7420 & 0.5330 & 0.5645 \\
\hline
\end{tabular}

(a) Primary vortex.

\begin{tabular}{lllll}
\hline Re & $\mathbf{1 0 0}$ & \multicolumn{3}{l}{$\mathbf{1 0 0 0}$} \\
\hline Right & $\mathbf{X}$ & $\mathbf{Y}$ & $\mathbf{X}$ & $\mathbf{Y}$ \\
\hline$[27]$ & 0.9375 & 0.0563 & 0.8625 & 0.1063 \\
{$[28]$} & 0.9453 & 0.0625 & 0.8594 & 0.1094 \\
{$[29]$} & 0.9451 & 0.0627 & 0.8667 & 0.1137 \\
{$[30]$} & 0.9417 & 0.0500 & 0.8643 & 0.1071 \\
{$[31]$} & 0.9375 & 0.0625 & 0.8625 & 0.1125 \\
Present & 0.9460 & 0.0570 & 0.8676 & 0.1126 \\
\hline
\end{tabular}

(b) Lower right vortex.

\begin{tabular}{|c|c|c|c|c|}
\hline $\mathbf{R e}$ & 100 & & 1000 & \\
\hline Left & $\mathbf{X}$ & $\mathbf{Y}$ & $\mathrm{X}$ & $\mathbf{Y}$ \\
\hline [27] & 0.0375 & 0.0313 & 0.0750 & 0.0813 \\
\hline [28] & 0.0313 & 0.0391 & 0.0859 & 0.0781 \\
\hline [29] & 0.0392 & 0.0353 & 0.0902 & 0.0784 \\
\hline [30] & 0.0333 & 0.0250 & 0.0858 & 0.0714 \\
\hline [31] & 0.0375 & 0.0375 & 0.0875 & 0.0750 \\
\hline Present & 0.0330 & 0.0332 & 0.0820 & 0.0756 \\
\hline
\end{tabular}

(c) Lower left vortex.

As the table shows, the predicted vortices locations are in a perfect match to the published results using other numerical methods which confirms the accuracy of the used MRT LBM.

\section{Conclusion}

The accuracy of the Multiple-relaxation-time Lattice Boltzmann Method for the simulation of incompressible viscous circulating flow is assessed. The method results are compared against published results for some benchmark problems. The lid-driven cavity flow is chosen as the benchmark case for circulating flow. The MR TLBM is used to model the 2D lid-driven cavity flow for a range of Reynolds numbers between 100 and 1000. The simulation results included the velocity profiles of the horizontal and vertical velocity components at different sections inside the cavity. The streamlines are also presented for a number of cases. The location of the primary central vortex in addition to the two side vortices are also calculated and compared to the published results. There is a perfect match between the results of the MRT LBM and the published ones. This confirms the ability and accuracy of the MRT LBM in simulating circulating flows.

\section{References}

[1] Witherden, F. D. and A. Jameson. Future Directions of Computational Fluid Dynamics. In $23^{\text {rd }}$ AIAA Computational Fluid Dynamics Conference. 2017.

[2] Li, Y., et al., Coupled computational fluid dynamics/multibody dynamics method for wind turbine aeroservo-elastic simulation including drive train dynamics. Renewable Energy, 2017.101: p. 1037-1051.

[3] Rutkowski, D. R., et al., Surgical planning for living donor liver transplant using 4D flow MRI, computational fluid dynamics and in vitro experiments. Computer Methods in Biomechanics and Biomedical Engineering: Imaging \& Visualization, 2017: p.1-11. 
[4] Fortunato, L., et al., In-situ assessment of biofilm formation in submerged membrane system using optical coherence tomography and computational fluid dynamics. Journal of Membrane Science, 2017.521: p.84-94.

[5] Boulard, T., et al., Modelling of micro meteorology, canopy transpiration and photosynthesis in a closed green house using computational fluid dynamics. Biosystems Engineering, 2017. 158: p.110-133.

[6] Tao, Y., K. Inthavong, and J. Tu, Computational fluid dynamics study of human-induced wake and particle dispersion in indoor environment. Indoor and Built Environment, 2017.26(2): p.185-198.

[7] Chen, S. and G. D. Doolen, LATTICE BOLTZMANN METHOD FOR FLUID FLOWS. Annual Review of Fluid Mechanics, 1998.30(1): p.329-364.

[8] Perumal, D. A. and A. K. Dass, Application of Lattice Boltzmann method for incompressible viscous flows. Applied Mathematical Modelling, 2013.37(6): p.4075-4092.

[9] He, Y., et al., Lattice Boltzmann method and its applications in engineering thermophysics. Chinese Science Bulletin, 2009.54(22): p.4117.

[10] Li, Q., et al., Lattice Boltzmann methods for multiphase flowand phase-change heat transfer. Progress in Energy and Combustion Science, 2016.52: p.62-105.

[11] Bao, J. and L. Schaefer, Lattice Boltzmann equation model for multi-component multi-phase flow with high density ratios. Applied Mathematical Modelling, 2013.37(4): p.1860-1871.

[12] Psihogios, J., et al., A Lattice Boltzmann study of nonnewtonian flow in digitally reconstructed porous domains. Transport in Porous Media, 2007.70(2): p.279-292.

[13] Wang, C.-H. and J.-R. Ho, A lattice Boltzmann approach for the non-Newtonian effect in the blood flow. Computers \& Mathematics with Applications, 2011.62(1): p.75-86.

[14] Hao, J. and L. Zhu, A lattice Boltzmann based implicit immersed boundary method for fluid-structure interaction. Computers \& Mathematics with Applications, 2010.59(1): p.185-193.

[15] Yang, J. and E. S. Boek, A comparison study of multicomponent Lattice Boltzmann models for flow in porous media applications. Computers \& Mathematics with Applications, 2013.65(6): p.882-890.

[16] Fakhari, A., D. Bolster, and L.-S. Luo, A weighted multiplerelaxation-time lattice Boltzmann method for multiphase flows and its application to partial coalescence cascades. Journal of Computational Physics, 2017.341: p.22-43.

[17] Zhuo, C. and P. Sagaut, Acoustic multipole sources for the regularized Lattice Boltzmann method: Comparison with multiple-relaxation-time models in the inviscid limit. Physical Review E, 2017.95(6): p.063301.
[18] Hu, Y., et al., A multiple-relaxation-time lattice Boltzmann model for the flow and heat transfer in a hydrodynamically and thermally anisotropic porous medium. International Journal of Heat and Mass Transfer, 2017.104: p.544-558.

[19] Liu, Q., Y.-L. He, and Q. Li, Enthalpy-based multiplerelaxation-time lattice Boltzmann method for solid-liquid phase-change heat transfer in metal foams. Physical Review E, 2017.96(2): p.023303.

[20] Mahmood, R., et al., Numerical Simulations of the Square Lid Driven Cavity Flow of Bingham Fluids Using Nonconforming Finite Elements Coupled with a Direct Solver. Advances in Mathematical Physics, 2017.2017.

[21] Abu-Nada, E. and A. J. Chamkha, Mixed convection flow of a nanofluid in a lid-driven cavity with a wavy wall. International Communications in Heat and Mass Transfer, 2014.57: p.36-47.

[22] Botella, O. and R. Peyret, Benchmark spectral results on the lid-driven cavity flow. Computers \& Fluids, 1998.27(4): p.421-433.

[23] Grillet, A. M., et al., Modeling of viscoelastic lid driven cavity flow using finite element simulations. Journal of NonNewtonian Fluid Mechanics, 1999.88(1): p.99-131.

[24] Benyahia, S., et al., Simulation of particles and gas flow behavior in the riser section of a circulating fluidized bed using the kinetic theory approach for the particulate phase. Powder Technology, 2000.112(1): p.24-33.

[25] Alex, J., et al., Analysis and design of suitable model structures for activated sludge tanks with circulating flow. Water science and technology, 1999.39(4): p.55-60.

[26] Bhatnagar, P. L., E. P. Gross, and M. Krook, A model for collision processes in gases. I. Small amplitude processes in charged and neutral one-component systems. Physical review, 1954.94(3): p.511.

[27] Vanka, S. P., Block-implicit multigrid solution of NavierStokes equations in primitive variables. Journal of Computational Physics, 1986.65(1): p.138-158.

[28] Ghia, U., K. N. Ghia, and C. T. Shin, High-Resolutions for incompressible flow using the Navier-Stokes equations and a multigrid method. Journal of Computational Physics, 1982.48(3): p.387-411.

[29] Hou, S., et al., Simulation of Cavity Flow by the Lattice Boltzmann Method. Journal of Computational Physics, 1995.118(2): p.329-347.

[30] Schreiber, R. and H. B. Keller, Driven cavity flows by efficient numerical techniques. Journal of Computational Physics, 1983.49(2): p.310-333.

[31] Gupta, M. M. and J. C. Kalita, A new paradigm for solving Navier-Stokes equations: stream function-velocity formulation. Journal of Computational Physics, 2005.207(1): p. $52-68$. 